\title{
Self-similar cosmologies in 5D: spatially flat anisotropic models
}

\author{
J. Ponce de Leon* \\ Laboratory of Theoretical Physics, Department of Physics \\ University of Puerto Rico, P.O. Box 23343, San Juan, \\ PR 00931, USA
}

July 2008

\begin{abstract}
In the context of theories of Kaluza-Klein type, with a large extra dimension, we study self-similar cosmological models in $5 D$ that are homogeneous, anisotropic and spatially flat. The "ladder" to go between the physics in $5 D$ and $4 D$ is provided by Campbell-Maagard's embedding theorems. We show that the 5 -dimensional field equations $R_{A B}=0$ determine the form of the similarity variable. There are three different possibilities: homothetic, conformal and "wave-like" solutions in $5 D$. We derive the most general homothetic and conformal solutions to the $5 D$ field equations. They require the extra dimension to be spacelike, and are given in terms of one arbitrary function of the similarity variable and three parameters. The Riemann tensor in $5 D$ is not zero, except in the isotropic limit, which corresponds to the case where the parameters are equal to each other. The solutions can be used as $5 D$ embeddings for a great variety of $4 D$ homogeneous cosmological models, with and without matter, including the Kasner universe. Since the extra dimension is spacelike, the $5 D$ solutions are invariant under the exchange of spatial coordinates. Therefore they also embed a family of spatially inhomogeneous models in $4 D$. We show that these models can be interpreted as vacuum solutions in braneworld theory. Our work (I) generalizes the $5 D$ embeddings used for the FLRW models; (II) shows that anisotropic cosmologies are, in general, curved in $5 D$, in contrast with FLRW models which can always be embedded in a $5 D$ Riemann-flat (Minkowski) manifold; (III) reveals that anisotropic cosmologies can be curved and devoid of matter, both in $5 D$ and $4 D$, even when the metric in $5 D$ explicitly depends on the extra coordinate, which is quite different from the isotropic case.
\end{abstract}

PACS: 04.50.+h; 04.20.Cv

Keywords: Kaluza-Klein Theory; Space-Time-Matter Theory; Braneworld Theory; General Relativity; Exact Solutions.

*E-mail: jpdel@ltp.upr.clu.edu or jpdel1@hotmail.com 


\section{Introduction}

In standard general relativity, exact solutions have played a crucial role in the development of many areas of astrophysics and cosmology [1, 2]. Exact solutions (i) provide a route to better and deeper understanding of gravity by giving non-perturbative insight into the highly nonlinear gravitational phenomena, (ii) can reveal unforeseen features of the theory that might be relevant to more general situations, and (iii) can be used for checking computer codes, which is important for the advent of numerical relativity [3].

In recent years there has been an increased interest in theories that envision our world as embedded in a universe with more than four large extra dimensions. Besides the longstanding theoretical motivations - as, e.g., resolving the differences between gravity and quantum field theory, and unifying all forces of nature - the interest in these theories has now a practical stimulus. Namely, that any solution of the N-dimensional Einstein equations with source can be locally embedded in a $(N+1)$-dimensional Ricci-flat manifold, which is guaranteed by Campbell-Maagard's embbedings theorems 4-7. In particular, this means that any solution of the $4 D$ Einstein equations with matter $G_{\mu \nu}=8 \pi T_{\mu \nu}$ (throughout the paper we set $c=G=1$ ) can always be locally embedded in a solution of the vacuum Einstein field equations $R_{A B}=0$ in $5 D$.

Finding an embedding for a particular $4 D$ spacetime, with a specified physical energy-momentum tensor, is an interesting and important question. However, it faces a fundamental problem. Namely, that the effective $4 D$ equations for gravity contain a source term, that is the spacetime projection of the $5 D$ Weyl tensor, which is unknown without specifying the properties of the metric in $5 D[8$. Therefore, the best way of deriving some practical benefit from Campbell-Maagard's theorem is by solving the equations in $5 D$, under some general geometrical properties, and then study the possible interpretations as well as the characteristics of the matter sources in $4 D$ that can generate these properties.

From a single solution in $5 D$ one may construct a great variety of scenarios in $4 D$ ranging from static configurations to cosmological solutions 9, which reflects the fact that (a) there are many ways of embedding a $4 D$ spacetime in a given higher-dimensional manifold, and (b) that the effective equations is $4 D$ do not form a closed system of equations. Therefore, the study of exact solutions of $5 D$ vacuum Einstein's equations is essential; not only because of the arguments (i)-(iii) given above, and for the formal mathematical aspects associated with the theory (e.g., the classification of $5 D$ manifolds) but also, for the growing importance of the application of Kaluza-Klein to cosmological and astrophysical phenomena in $4 D$. The study of all possible scenarios is important in order to be able to predict observable effects in $4 D$ caused by new physics from an extra dimension.

Nearly all the solutions in $5 D$ are obtained under the assumption of spatial spherical symmetry and the choice of one or more additional functional relations that simplify the field equations in such a way that they become fully integrable. The disadvantage of this approach is that different choices may lead to the same physical solution, in different disguise.

In a recent work [10] we started a systematic investigation of the solutions of the $5 D$ field equations $R_{A B}=0$ for the case where the $5 D$ metric possesses self-similar symmetry. We found the most general self-similar, homogeneous and isotropic, Ricci-flat cosmologies in $5 D$ and showed that they can be interpreted, or used, as $5 D$ Riemann-flat embeddings for spatially flat FRW cosmologies in $4 D$. In this paper we continue that investigation, but we abandon the assumption of perfect spatial isotropy and consider cosmologies with spatial anisotropy.

For the models to be physically realistic they have to closely resemble the standard FRW models. It is well known that, within the context of Bianchi classification, the generalization of the "flat", "open" and "closed" FRW models are cosmologies of Bianchi type I, V and IX, respectively [11. Cosmological observations today indicate that our universe is spatially flat [12]-14. Thus, Bianchi I models are the closest anisotropic approximation to the present stage of evolution of our universe.

Consequently, in this paper we adopt the 5 -dimensional line element 1

$$
d S^{2}=e^{\nu(t, \psi)} d t^{2}-e^{\lambda(t, \psi)} d x^{2}-e^{\mu(t, \psi)} d y^{2}-e^{\sigma(t, \psi)} d z^{2}+\epsilon e^{\omega(t, \psi)} d \psi^{2},
$$

which on every hypersurface $\psi=$ constant, reduces to homogeneous and anisotropic Bianchi type-I cosmological

\footnotetext{
${ }^{1}$ In this work we use the following conventions: $t=x^{0}, x=x^{1}, y=x^{2}$ and $z=x^{3}$ are the usual spacetime coordinates; $\psi=x^{4}$ represents the coordinate along the "extra" dimension; the signature of the $5 D$ metric is $(+,-,-,-, \epsilon)$ where $\epsilon$ can be either -1 or +1 depending on whether the extra dimension is spacelike or timelike. The range of tensor indices is $A, B \ldots=0-4$ and $\mu, \nu, \ldots=0-3$.
} 
models with flat spatial sections. A simplified version of this metric has been studied by Roque and Seiler [15] under the assumption of separability. In this work we obtain the general solutions to the field equations $R_{A B}=0$ under the assumption that the metric (1) possesses self-similar symmetry. This assumption is motivated not only by the fact that FRW models are self-similar in $4 D[16$ and $5 D[10$, but also by the "similarity hypothesis" of Carr and Coley [17, which asserts that under a variety of physical circumstances, both homogeneous and inhomogeneous cosmological models (in $4 D$ ) naturally evolve to a self-similar form2 2

We demonstrate that the field equations in $5 D$ allow three types of similarity solutions: homothetic, conformal and "wave-like". We show how to integrate the field equations for the first two types of similarity. We find that the solutions (a) depend on one arbitrary function and three arbitrary parameters; (b) require the extra dimension to be spacelike, and (c) generalize the family of homogeneous and isotropic $5 D$ models discussed in [10. In accordance with Campbell-Maagard's theorem they can be used, or interpreted, as $5 D$ embeddings for four-dimensional subspaces. Depending on how we do the actual embedding, one can construct $4 D$ anisotropic cosmological models that are either homogeneous or inhomogeneous, although homogeneous isotropic cosmologies are recovered as particular cases.

By means of dimensional reduction of the metric in $5 D$, we analyze, in some detail, possible applications of the family of homothetic solutions in $5 D$. We find that all the models constructed in $4 D$, exhibit some type of selfsimilarity. Those that are inhomogeneous and anisotropic inherit the homothetic symmetry from the $5 D$ embedding. The rest of them are either partially homothetic or homothetic along some $4 D$ vector. Thus, all these models represent different self-similar scenarios in $4 D$ and, as such, can be relevant to the similarity hypothesis mentioned above.

The paper is organized as follows. In section 2 , we deduce the shape of the similarity variable. In section 3 , we solve the field equations with homothetic and conformal symmetry in $5 D$. In section 4 , we present some general properties of the solutions in $5 D$. In section 5 we show different admissible interpretations in $4 D$, but an exhaustive treatment of all possibilities is beyond the scope of this work. In section 6 we give a summary.

\section{Similarity variable}

In a self-similar model, by a suitable transformation of coordinates all the dimensionless quantities can be put in a form where they are functions only of a single variable (say $\zeta$ ) 22]-29]. Thus, in the case under consideration, in "self-similar" coordinates $(\bar{t}, \bar{x}, \bar{y}, \bar{z}, \bar{\psi})$, the line element (10) can be written as

$$
d S^{2}=e^{\nu(\zeta)} d \bar{t}^{2}-e^{\lambda(\zeta)} d \bar{x}^{2}-e^{\mu(\zeta)} d \bar{y}^{2}-e^{\sigma(\zeta)} d \bar{z}^{2}+\epsilon e^{\omega(\zeta)} d \bar{\psi}^{2}
$$

where $\zeta$ is some function of $\bar{t}$ and $\bar{\psi}$, viz.,

$$
\zeta=\zeta(\bar{t}, \bar{\psi})
$$

The metric functions $\nu(\zeta), \lambda(\zeta), \mu(\zeta), \sigma(\zeta), \omega(\zeta)$, as well as $\zeta$ and the signature coefficient $\epsilon$, have to satisfy the field equations $R_{A B}=0$. In order to avoid misunderstanding, it is worth mentioning that in the literature the concept of self-similarity is frequently equated with homothetic symmetry. In this work we follow the traditional nomenclature used in 22]-24]. Among other things, we will see that the self-similar line element (2) does not necessarily admit a homothetic Killing vector. In what follows we are going to suppress the bar over the self-similar coordinates.

The shape of the similarity variable $\zeta$ is determined by the field equations. Firstly, we note that from $R_{04}=0$ it follows that

$$
2\left(\lambda_{\zeta}+\mu_{\zeta}+\sigma_{\zeta}\right) \dot{\zeta}^{\prime}+\left[\left(\lambda_{\zeta}^{2}+\mu_{\zeta}^{2}+\sigma_{\zeta}^{2}\right)-\left(\lambda_{\zeta}+\mu_{\zeta}+\sigma_{\zeta}\right)\left(\nu_{\zeta}+\omega_{\zeta}\right)+2\left(\lambda_{\zeta \zeta}+\mu_{\zeta \zeta}+\sigma_{\zeta \zeta}\right)\right] \zeta^{\prime} \dot{\zeta}=0,
$$

where $f_{\zeta}$ denotes derivative of $f$ with respect to $\zeta$; dots and primes stand for derivatives with respect to $t$ and $\psi$, respectively.

\footnotetext{
${ }^{2}$ Although there are some counterexamples to this hypothesis [18, 19, there is a strong evidence that self-similar models play a significant role at asymptotic regimes [20-21].
} 


\subsection{Homothetic and conformal self-similar variable}

Equation (4) implies that, in the general case where $\dot{\zeta}^{\prime} \neq 0$, the assumed self-similarity requires that the ratio $\left[\dot{\zeta}^{\prime} /\left(\zeta^{\prime} \dot{\zeta}\right)\right]$ be some function of $\zeta$. Clearly, any separable function of $t$ and $\psi$ will do the job. Therefore, without loss of generality we can set

$$
\zeta=\frac{T(t)}{F(\psi)}
$$

where $T$ and $F$ are some functions of their arguments. Consequently, the equations $R_{00}=0, R_{11}=0, R_{22}=0, R_{33}=$ 0 and $R_{44}=0$ they all have the structure

$$
\left(\frac{\dot{T}^{2}}{F^{\prime 2}}\right) M(\zeta)+\left(\frac{\ddot{T} F}{F^{\prime 2}}\right) N(\zeta)+\left(2-\frac{F F^{\prime \prime}}{F^{\prime 2}}\right) P(\zeta)+Q(\zeta)=0
$$

where $M, N, P$ and $Q$ symbolize the corresponding functions of $\zeta$ in these equations. Therefore, in order to preserve the self-similar symmetry, we have to require

$$
2-\frac{F F^{\prime \prime}}{F^{\prime 2}}=l
$$

where $l$ is a dimensionless separation constant. Integrating this expression we obtain

$$
F^{\prime}=p F^{(2-l)},
$$

where $p$ is a constant of integration. Thus, we find

$$
\left(\frac{\dot{T}^{2}}{F^{\prime 2}}\right)=\left[\frac{\dot{T}^{2}}{p^{2} T^{(4-2 l)}}\right] \zeta^{(4-2 l)}, \text { and }\left(\frac{\ddot{T} F}{F^{\prime 2}}\right)=\left[\frac{\ddot{T}}{p^{2} T^{(3-2 l)}}\right] \zeta^{(3-2 l)} .
$$

Consistency of (6) demands the quantities inside the square brackets to be constants, which requires

$$
T \sim\left\{\begin{array}{cc}
t^{1 /(l-1)} & \text { for } l \neq 1, \\
e^{q t} & \text { for } l=1,
\end{array}\right.
$$

where $q$ is some constant 3 . Consequently, without loss of generality we can set

$$
\zeta=\left\{\begin{array}{cc}
\left(\frac{t}{\psi}\right)^{1 /(l-1)} & \text { for } l \neq 1, \\
\left(\frac{e^{q t}}{e^{p \psi}}\right) & \text { for } l=1 .
\end{array}\right.
$$

We will see that the variables $\zeta$ for $l \neq 1$ and $l=1$ lead to homothetic and conformal solutions in $5 D$, respectively.

\subsection{Wave-like self-similar variable}

Finally, in the particular case where $\dot{\zeta}^{\prime}=0$, it is not difficult to show that the similarity variable must have the form

$$
\zeta=\omega_{0} t+k_{0} \psi
$$

where $\omega_{0}$ and $k_{0}$ are some constants with the appropriate units. Thus, in this case the metric functions have a dependence of time and the extra coordinate like in traveling waves or pulses propagating along the fifth dimension. Planes waves or wave-like solutions in $5 D$, in the case of spatial spherical symmetry, have been studied by Wesson, Liu and Seahra [30], 31]; Horowitz, Low and Zee [32]; as well as by the present author 33.

\footnotetext{
${ }^{3}$ We note that $p$ and $q$ must have the units of (length) ${ }^{-1}$.
} 


\section{$3 \quad$ Field equations}

The field equations $R_{A B}=0$ look quite different for $\dot{\zeta}^{\prime} \neq 0$ and $\dot{\zeta}^{\prime}=0$. In what follows we will consider the general case where $\dot{\zeta}^{\prime} \neq 0$, for which the metric functions depend on (11). Wave-like solutions will be considered elsewhere.

Let us notice that for $l=2$ the similarity variable becomes $\zeta_{(l=2)} \equiv \xi=(t / \psi)$. Thus, for an arbitrary $l \neq 1$ we can write $\zeta=\xi^{1 /(l-1)}$. What this means is that we can first study the integration of the equations for $l=2$, and then obtain the solution for any value of $l$ by a simple transformation

$$
\xi \rightarrow \zeta^{l-1}, \quad \xi \equiv \frac{t}{\psi} .
$$

\subsection{Integrating the field equations for $l=2$}

The non-vanishing components of the Ricci tensor in $5 D$ are

1. $R_{00}=0$,

$$
\begin{array}{r}
\nu_{\xi}\left[\left(\epsilon e^{\omega}-\xi^{2} e^{\nu}\right)\left(\nu_{\xi}+\lambda_{\xi}+\mu_{\xi}+\sigma_{\xi}+\frac{2 \nu_{\xi \xi}}{\nu_{\xi}}\right)-4 \xi e^{\nu}+\omega_{\xi}\left(\epsilon e^{\omega}+\xi^{2} e^{\nu}\right)\right]= \\
\epsilon\left[\left(\nu_{\xi}^{2}+\lambda_{\xi}^{2}+\mu_{\xi}^{2}+\sigma_{\xi}^{2}+\omega_{\xi}^{2}\right)+2\left(\nu_{\xi \xi}+\lambda_{\xi \xi}+\mu_{\xi \xi}+\sigma_{\xi \xi}+\omega_{\xi \xi}\right)\right] e^{\omega} .
\end{array}
$$

2. $R_{11}=0$,

$$
\lambda_{\xi}\left[\left(\epsilon e^{\omega}+\xi^{2} e^{\nu}\right)\left(\lambda_{\xi}+\frac{2 \lambda_{\xi \xi}}{\lambda_{\xi}}+\mu_{\xi}+\sigma_{\xi}\right)+4 \xi e^{\nu}+\left(\epsilon e^{\omega}-\xi^{2} e^{\nu}\right)\left(\omega_{\xi}-\nu_{\xi}\right)\right]=0 .
$$

3. $R_{22}=0$,

$$
\mu_{\xi}\left[\left(\epsilon e^{\omega}+\xi^{2} e^{\nu}\right)\left(\mu_{\xi}+\frac{2 \mu_{\xi \xi}}{\mu_{\xi}}+\lambda_{\xi}+\sigma_{\xi}\right)+4 \xi e^{\nu}+\left(\epsilon e^{\omega}-\xi^{2} e^{\nu}\right)\left(\omega_{\xi}-\nu_{\xi}\right)\right]=0
$$

4. $R_{33}=0$,

$$
\sigma_{\xi}\left[\left(\epsilon e^{\omega}+\xi^{2} e^{\nu}\right)\left(\sigma_{\xi}+\frac{2 \sigma_{\xi \xi}}{\sigma_{\xi}}+\lambda_{\xi}+\mu_{\xi}\right)+4 \xi e^{\nu}+\left(\epsilon e^{\omega}-\xi^{2} e^{\nu}\right)\left(\omega_{\xi}-\nu_{\xi}\right)\right]=0 .
$$

5. $R_{44}=0$,

$$
\begin{array}{r}
\omega_{\xi}\left[\left(\epsilon e^{\omega}-\xi^{2} e^{\nu}\right)\left(\lambda_{\xi}+\mu_{\xi}+\sigma_{\xi}+\omega_{\xi}+\frac{2 \omega_{\xi \xi}}{\omega_{\xi}}\right)-\nu_{\xi}\left(\epsilon e^{\omega}+\xi^{2} e^{\nu}\right)-4 \xi e^{\nu}\right]= \\
-\xi\left[4\left(\nu_{\xi}+\lambda_{\xi}+\mu_{\xi}+\sigma_{\xi}+\omega_{\xi}\right)+\xi\left(\nu_{\xi}^{2}+\lambda_{\xi}^{2}+\mu_{\xi}^{2}+\sigma_{\xi}^{2}+\omega_{\xi}^{2}\right)+2 \xi\left(\nu_{\xi \xi}+\lambda_{\xi \xi}+\mu_{\xi \xi}+\sigma_{\xi \xi}+\omega_{\xi \xi}\right)\right] e^{\nu} .
\end{array}
$$

6. Finally, $R_{04}=0$,

$$
2 \xi\left(\lambda_{\xi \xi}+\nu_{\xi \xi}+\sigma_{\xi \xi}\right)+\left(2-\xi \nu_{\xi}-\xi \omega_{\xi}\right)\left(\lambda_{\xi}+\sigma_{\xi}+\mu_{\xi}\right)+\xi\left(\lambda_{\xi}^{2}+\mu_{\xi}^{2}+\sigma_{\xi}^{2}\right)=0 .
$$

We note that equations (15), (16) and (17) require

$$
\frac{\lambda_{\xi \xi}}{\lambda_{\xi}}=\frac{\mu_{\xi \xi}}{\mu_{\xi}}=\frac{\sigma_{\xi \xi}}{\sigma_{\xi}}
$$

Therefore, without loss of generality one can set

$$
e^{\lambda}=A f^{2 \alpha}(\xi) ; \quad e^{\mu}=B f^{2 \beta}(\xi), \quad e^{\sigma}=C f^{2 \gamma}(\xi),
$$

where $A, B, C$ are constants; $f$ is some function of the variable $\xi=(t / \psi)$; and $\alpha, \beta$ and $\gamma$ are arbitrary parameters. As a consequence, $R_{11}=0, R_{22}=0$ and $R_{33}=0$ reduce to 


$$
\left(\epsilon e^{\omega}+\xi^{2} e^{\nu}\right)\left[\frac{f_{\xi \xi}}{f_{\xi}}+\frac{f_{\xi}}{f}(\alpha+\beta+\gamma-1)\right]-\frac{1}{2}\left(\nu_{\xi}-\omega_{\xi}\right)\left(\epsilon e^{\omega}-\xi^{2} e^{\nu}\right)+2 \xi e^{\nu}=0 .
$$

On the other hand $R_{04}$ yields

$$
(\alpha+\beta+\gamma)\left(\frac{2 f_{\xi \xi}}{f_{\xi}}-\nu_{\xi}-\omega_{\xi}+\frac{2}{\xi}\right)+2\left(\alpha^{2}+\beta^{2}+\gamma^{2}-\alpha-\beta-\gamma\right)\left(\frac{f_{\xi}}{f}\right)=0
$$

from which we get

$$
e^{(\nu+\omega) / 2}=E \xi f^{(b / a)} f_{\xi}
$$

where $E$ is a constant of integration, and

$$
a \equiv(\alpha+\beta+\gamma), \quad b \equiv\left(\alpha^{2}+\beta^{2}+\gamma^{2}-\alpha-\beta-\gamma\right) .
$$

Clearly, from (24) it follows that there are different scenarios depending on whether $(\nu+\omega) \neq 0$ or $(\nu+\omega)=0$. We now proceed to discuss them separately.

\subsubsection{General solution for $l=2$}

For the general case where $\nu+\omega \neq 0$ in (24), we substitute $e^{\nu / 2}=E \xi f^{(b / a)} f_{\xi} e^{-\omega / 2}$ into (14) and (18). Since these are second order differential equations for $\nu$, we obtain two differential equations containing $f_{\xi \xi \xi}$, the third derivative of $f(\xi)$. Next, we isolate the $f_{\xi \xi \xi}$ obtained from $R_{00}=0$ and $R_{44}=0$, say $f_{\xi \xi \xi}\left(R_{00}\right)$ and $f_{\xi \xi \xi}\left(R_{44}\right)$, respectively. Then, from the compatibility condition

$$
f_{\xi \xi \xi}\left(R_{00}\right)=f_{\xi \xi \xi}\left(R_{44}\right)
$$

we find

$$
e^{2 \omega}=-\epsilon E^{2} \xi^{4} f^{(2 b / a)} f_{\xi}^{2}
$$

This expression demands the extra dimension $\psi$ to be spacelike, i.e., $\epsilon=-1$. In addition from (24) we find

$$
e^{\nu}=E f^{(b / a)} f_{\xi}, \quad \text { and } \quad e^{\omega(\xi)}=\xi^{2} e^{\nu(\xi)} .
$$

Now, it is easy to verify that the field equation (22) is identically satisfied for $e^{\omega}=\xi^{2} e^{\nu}$ and $\epsilon=-1$.

Collecting results, we have showed that the $5 D$ line element

$$
d S^{2}=E f^{(b / a)} f_{\xi} d t^{2}-A f^{2 \alpha} d x^{2}-B f^{2 \beta} d y^{2}-C f^{2 \gamma} d z^{2}-E \xi^{2} f^{(b / a)} f_{\xi} d \psi^{2},
$$

is the general solution of the field equations (14)-(19), for any arbitrary function $f=f(\xi)$ with $\xi=t / \psi$.

\subsubsection{Particular solution for $l=2$}

For the case where $\nu+\omega=0$, but $\nu=-\omega \neq 0$, integrating (24) we obtain the solution as follows

$$
d S^{2}=\left(\frac{1}{\xi}\right) d t^{2}-A h^{2 \alpha} d x^{2}-B h^{2 \beta} d y^{2}-C h^{2 \gamma} d z^{2}-\xi d \psi^{2}, \quad \text { with } h=\left[C_{1} \ln \xi+C_{2}\right]^{a /(a+b)},
$$

where $C_{1}$ and $C_{2}$ are constants of integration. It should be mentioned that this is the unique family of solutions with $\nu=-\omega \neq 0$.

In the case where $\nu=-\omega=0$ the field equations $R_{A B}=0$ require $a=\alpha+\beta+\gamma=0$, i.e., $h=1$. Thus, this case yields flat Minkowski space in $5 D$ and $4 D$. 


\subsection{Solutions for $l \neq 1$}

In order to construct the solutions with $l \neq 1$, we now use the transformation (13). The second equation in (28) becomes

$$
e^{\omega(\zeta)}=\zeta^{2(l-1)} e^{\nu(\zeta)}
$$

On the other hand $f_{\xi} \rightarrow \zeta^{2-l} f_{\zeta}$. Thus, from (29) we obtain the general solution for $l \neq 1$ as

$$
d S^{2}=\bar{E} \zeta^{2-l} f^{(b / a)} f_{\zeta} d t^{2}-A f^{2 \alpha} d x^{2}-B f^{2 \beta} d y^{2}-C f^{2 \gamma} d z^{2}-\bar{E} \zeta^{l} f^{(b / a)} f_{\zeta} d \psi^{2} .
$$

In addition, from (30) we get

$$
d S^{2}=\left(\frac{1}{\zeta^{l-1}}\right) d t^{2}-A h^{2 \alpha} d x^{2}-B h^{2 \beta} d y^{2}-C h^{2 \gamma} d z^{2}-\zeta^{l-1} d \psi^{2}, \quad \text { with } h \equiv\left[C_{1} \ln \zeta+C_{2}\right]^{a /(a+b)}
$$

which in terms of $t$ and $\psi$ is identical to (30). However, we put it here in explicit form in order to develop solutions with $l=1$.

\subsection{Solutions for $l=1$}

The field equations $R_{A B}=0$ require $p=q$. Therefore the similarity variable now becomes

$$
\zeta_{(l=1)} \equiv \tilde{\zeta}=\left(\frac{e^{t}}{e^{\psi}}\right)^{q}
$$

In this case the solutions are readily obtained from (32) and (33) just by setting $l=1$ and replacing $\zeta$ by $\tilde{\zeta}$. Thus, from (31) we get

$$
e^{\omega(\tilde{\zeta})}=e^{\nu(\tilde{\zeta})}
$$

Consequently, the general solution with $l=1$ is given by

$$
d S^{2}=E \tilde{\zeta} f^{(b / a)} f_{\tilde{\zeta}} d t^{2}-A f^{2 \alpha} d x^{2}-B f^{2 \beta} d y^{2}-C f^{2 \gamma} d z^{2}-E \tilde{\zeta} f^{(b / a)} f_{\tilde{\zeta}} d \psi^{2}
$$

Likewise, from (33) we get the particular solution

$$
d S^{2}=d t^{2}-A h^{2 \alpha} d x^{2}-B h^{2 \beta} d y^{2}-C h^{2 \gamma} d z^{2}-d \psi^{2}, \quad \text { with } \quad h \equiv\left[C_{1} \ln \tilde{\zeta}+C_{2}\right]^{a /(a+b)} .
$$

\subsection{Solutions for $a=\alpha+\beta+\gamma=0$}

It should be noted that (29), (32) and (36) require $a=(\alpha+\beta+\gamma) \neq 0$. If $a=0$, then from (23) it follows that either $b=0$, or $f=$ constant. In both cases the $5 D$ metric becomes

$$
d S^{2}=e^{\nu(\zeta)} d t^{2}-d x^{2}-d y^{2}-d z^{2}+\epsilon e^{\omega(\zeta)} d \psi^{2}
$$

At this point, it is crucial to emphasize that (31) and (35) hold for any particular value of $\alpha, \beta$ and $\gamma$. Therefore, the line elements

$$
d S^{2}=e^{\nu(\zeta)} d t^{2}-d x^{2}-d y^{2}-d z^{2}-\zeta^{2(l-1)} e^{\nu(\zeta)} d \psi^{2}, \quad \zeta=\left(\frac{t}{\psi}\right)^{1 /(l-1)}
$$

and

$$
d S^{2}=e^{\nu(\tilde{\zeta})} d t^{2}-d x^{2}-d y^{2}-d z^{2}-e^{\nu(\tilde{\zeta})} d \psi^{2}, \quad \tilde{\zeta}=\left(\frac{e^{t}}{e^{\psi}}\right)^{q}
$$

constitute the most general solutions of the field equations $R_{A B}=0$, for the metric (38). We also note that (30), (33) and (37) require $(a+b)=\alpha^{2}+\beta^{2}+\gamma^{2} \neq 0$. This is always satisfied unless $\alpha=\beta=\gamma=0$, in which case the line element has the form (38). 


\section{Some properties of the solutions}

The general solutions (32) and (36) depend on one arbitrary function, of the corresponding similarity variable, and contain three arbitrary parameters $\alpha, \beta$ and $\gamma$. In addition, the field equations require the extra dimension to be spacelike $(\epsilon=-1)$. For $\alpha=\beta=\gamma$ we recover cosmological models with spatial spherical symmetry. Indeed, setting $f^{2 \alpha}=f^{2 \beta}=f^{2 \gamma}=e^{\lambda(\xi)}$; changing coordinates: $x=r \sin \theta \cos \phi, y=r \sin \theta \sin \phi, z=r \cos \theta$; re-naming the constants, without loss of generality (32) can be written as

$$
d S^{2}=\left(\frac{1}{C}\right) \zeta^{(2-l)} \lambda_{\zeta} e^{\lambda(\zeta) / 2} d t^{2}-e^{\lambda(\zeta)}\left[d r^{2}+r^{2}\left(d \theta^{2}+\sin ^{2} \theta d \phi^{2}\right)\right]-\left(\frac{1}{C}\right) \zeta^{l} \lambda_{\zeta} e^{\lambda(\zeta) / 2} d \psi^{2} .
$$

Likewise (36) becomes

$$
d S^{2}=\left(\frac{1}{C}\right) \tilde{\zeta} \lambda \tilde{\zeta} e^{\lambda(\tilde{\zeta}) / 2} d t^{2}-e^{\lambda(\tilde{\zeta})}\left[d r^{2}+r^{2}\left(d \theta^{2}+\sin ^{2} \theta d \phi^{2}\right)\right]-\left(\frac{1}{C}\right) \tilde{\zeta} \lambda \tilde{\zeta} e^{\lambda(\tilde{\zeta}) / 2} d \psi^{2} .
$$

These two metrics represent the most general self-similar, homogeneous and isotropic, Ricci-flat cosmologies in $5 D$ [10.

Although solutions (36) are formally obtained from (32) just by setting $l=1$, they have different geometrical properties. In particular, solutions (32) admit a homothetic Killing vector in $5 D$ for any values of $\alpha, \beta$ and $\gamma$, namely,

$$
\mathcal{L}_{\zeta} g_{A B}^{(l \neq 1)}=2 g_{A B}^{(l \neq 1)}, \quad \text { with } \quad \zeta_{(l \neq 1)}^{C}=(t, x, y, z, \psi),
$$

where $g_{A B}^{(l \neq 1)}$ is the metric (32) and $\mathcal{L}_{\zeta}$ denotes the Lie derivative along the $5 D$ vector $\zeta_{(l \neq 1)}^{C}$. On the other hand, solutions (36) are self-similar but do not admit a homothetic Killing vector, except for $\alpha=\beta=\gamma$. In general, they admit an infinitesimal conformal transformation parameterized by some function $H=H(t+\psi)$, viz.,

$$
\mathcal{L}_{\zeta} g_{A B}^{(l=1)}=2 \dot{H} g_{A B}^{(l=1)}, \quad \text { with } \zeta_{(l=1)}^{C}=(H, x \dot{H}, y \dot{H}, z \dot{H}, H), \quad \text { and } \quad H=H(t+\psi) .
$$

Another difference is that (32) is invariant under the transformation: $t \rightarrow i \psi, \psi \rightarrow i t$ (double Wick rotations) and $\bar{E} \rightarrow-\hat{E}$. Indeed, it is easy to verify that

$$
d S^{2}=\hat{E} \hat{\zeta}^{l} f^{(b / a)} f_{\hat{\zeta}} d t^{2}-A f^{2 \alpha} d x^{2}-B f^{2 \beta} d y^{2}-C f^{2 \gamma} d z^{2}-\hat{E} \hat{\zeta}^{(2-l)} f^{(b / a)} f_{\hat{\zeta}} d \psi^{2}, \quad \text { with } \quad \hat{\zeta} \equiv \frac{1}{\zeta}
$$

is also a solution of the field equations. The same occurs with (36) but the metric functions in the "Wick rotated" line element are now complex functions.

However, the solutions share some important properties. For example, one can show that there are only two cases where the components of the $5 D$ Riemann tensor vanish: (i) $f=$ constant (or $\alpha=\beta=\gamma=0$ ), and (ii) $\alpha=\beta=\gamma \neq 0$. In the first case the line element and the metric functions are given by (38). In the second case the spatial sections $t=$ constant and $\psi=$ constant are flat and possess spherical symmetry [10. In any other circumstance, the manifold is curved in $5 D$. As an illustration we present here the Riemann tensor in $5 D$, calculated with the line element (29),

$$
\begin{aligned}
& R_{0114}=\xi R_{0101}=\frac{R_{1414}}{\xi}=-\frac{A \alpha f^{2(\alpha-1)} \xi f_{\xi}^{2}\left[\beta^{2}+\gamma^{2}-\alpha(\beta+\gamma)\right]}{a \psi^{2}}, \\
& R_{0224}=\xi R_{0202}=\frac{R_{2424}}{\xi}=-\frac{B \beta f^{2(\beta-1)} \xi f_{\xi}^{2}\left[\alpha^{2}+\gamma^{2}-\beta(\alpha+\gamma)\right]}{a \psi^{2}}, \\
& R_{0334}=\xi R_{0303}=\frac{R_{3434}}{\xi}=-\frac{C \gamma f^{2(\gamma-1)} \xi f_{\xi}^{2}\left[\alpha^{2}+\beta^{2}-\gamma(\alpha+\beta)\right]}{a \psi^{2}} .
\end{aligned}
$$

Finally, it is essential to mention that the solutions discussed in the preceding section are invariant under the change $(x, y, z) \leftrightarrow \psi$, which is a consequence of the fact that the extra coordinate $\psi$ is spacelike. For example, if we change $\psi \leftrightarrow z$ and denote

$$
F \equiv F(\eta), \quad \text { with } \quad \eta=\frac{t}{z},
$$


then the line element

$$
d S^{2}=E F^{(b / a)} F_{\eta}\left(d t^{2}-\eta^{2} d z^{2}\right)-A F^{2 \alpha} d x^{2}-B F^{2 \beta} d y^{2}-C F^{2 \gamma} d \psi^{2},
$$

which we have constructed from (29), is also a solution of the field equations $R_{A B}=0$. Certainly, the same is true for all solutions discussed in section 3. It is important to note that the metrics obtained under the change $(x, y, z) \leftrightarrow \psi$ (i) are independent on the "extra" dimension $\psi$, and (ii) the spatial sections defined by $t=$ constant, $\psi=$ constant are non-flat. In the next section we will see that this symmetry is crucial for the interpretation of the solutions in $4 D$.

\section{Interpretation in $4 D$}

We have already mentioned that there are different ways of producing, or embedding, a $4 D$ spacetime in a given higher-dimensional manifold. However, the most popular approach is based on three different assumptions. First, that we can use the coordinate frame 34. Second, that our $4 D$ spacetime can be recovered by going onto a hypersurface $\Sigma_{\psi}: \psi=\psi_{0}=$ constant, which is orthogonal to the $5 D$ unit vector

$$
\hat{n}^{A}=\frac{\delta_{4}^{A}}{\sqrt{\epsilon g_{44}}}, \quad n_{A} n^{A}=\epsilon,
$$

along the extra dimension. Third, that the physical metric of the spacetime can be identified with the one induced on $\Sigma_{\psi}$.

For a line element of the form

$$
d S^{2}=g_{\mu \nu}\left(x^{\rho}, \psi\right) d x^{\mu} d x^{\nu}+\epsilon \Phi^{2}\left(x^{\rho}, \psi\right) d \psi^{2},
$$

the induced metric on hypersurfaces $\Sigma_{\psi}$ is just $g_{\mu \nu}$, i.e., the $4 D$ part of the metric in $5 D$. The crucial moment is that, although the energy-momentum tensor (EMT) in $5 D$ is zero, to an observer confined to making physical measurements in our ordinary spacetime, and not aware of the extra dimension, the spacetime is not empty but contains (effective) matter whose EMT, ${ }^{(4)} T_{\alpha \beta}$, is determined by the Einstein equations in $4 D$, namely

$$
{ }^{(4)} G_{\alpha \beta}=8 \pi^{(4)} T_{\alpha \beta}=-\epsilon\left(K_{\alpha \lambda} K_{\beta}^{\lambda}-K_{\lambda}^{\lambda} K_{\alpha \beta}\right)+\frac{\epsilon}{2} g_{\alpha \beta}\left(K_{\lambda \rho} K^{\lambda \rho}-\left(K_{\lambda}^{\lambda}\right)^{2}\right)-\epsilon E_{\alpha \beta},
$$

where $K_{\mu \nu}$ is the extrinsic curvature

$$
K_{\alpha \beta}=\frac{1}{2} \mathcal{L}_{\hat{n}} g_{\alpha \beta}=\frac{1}{2 \Phi} \frac{\partial g_{\alpha \beta}}{\partial \psi}
$$

$E_{\mu \nu}$ is the projection of the bulk Weyl tensor ${ }^{(5)} C_{A B C D}$ orthogonal to $\hat{n}^{A}$, i.e., "parallel" to spacetime, viz.,

$$
E_{\alpha \beta}={ }^{(5)} C_{\alpha A \beta B} \hat{n}^{A} \hat{n}^{B}=-\frac{1}{\Phi} \frac{\partial K_{\alpha \beta}}{\partial \psi}+K_{\alpha \rho} K_{\beta}^{\rho}-\epsilon \frac{\Phi_{\alpha ; \beta}}{\Phi},
$$

and $\Phi_{\alpha} \equiv \partial \Phi / \partial x^{\alpha}$. It is important to mention that the effective matter content of the spacetime is the same whether we interpret it in space-time-matter theory [35]-[36, or in a $\mathbf{Z}_{2}$ symmetric brane universe [37. Indeed, these two theories are mathematically equivalent, although they have different motivation and physical interpretation [38.

\subsection{Homogeneous cosmological models}

Since $\zeta=\xi^{1 /(l-1)}$, it follows that the models with $l \neq 1$, discussed in Section 3.1, are physically indistinguishable from those with $l=2$. The only reason for considering them here is a mathematical one: they allow us to generate non-homothetic $5 D$ models $(l=1)$ from homothetic ones $(l \neq 1)$. In what remains we focus our attention to metrics (29) and (48), which admit a homothetic Killing vector in $5 D$, namely (43), and therefore are relevant to the similarity hypothesis mentioned in the Introduction 4 .

\footnotetext{
${ }^{4}$ We shall come back to discuss the 4-dimensional interpretation of the non-homothetic $5 D$ models $(l=1)$ elsewhere.
} 
However, by virtue of the symmetry of the solutions in $5 D$, which in the case under consideration is expressed by (45) and (48), there are a number of distinct possible scenarios in $4 D$. An exhaustive treatment of all these scenarios is beyond the purview of this work. Rather, in this section we restrict ourselves to presenting some representative models.

In the approach under consideration, the spacetime metric generated by (29) is given by

$$
d s^{2} \equiv d S_{\mid \Sigma_{\psi}}^{2}=g_{\mu \nu} d x^{\mu} d x^{\nu}=E f^{(b / a)} f_{\xi} d t^{2}-A f^{2 \alpha} d x^{2}-B f^{2 \beta} d y^{2}-C f^{2 \gamma} d z^{2} .
$$

Let us emphasize that, on each hypersurface $\Sigma_{\psi}$, this metric is a function of time only. Therefore, (54) yields homogeneous, anisotropic cosmological models of Bianchi type I.

A general feature of metrics (54) is that they are partially homothetic, i.e.,

$$
\mathcal{L}_{\zeta} g_{00}=0, \quad \mathcal{L}_{\zeta} g_{i j}=2 g_{i j}, \quad \text { with } \quad \zeta^{\mu}=(0, x, y, z),
$$

for any arbitrary function $f$.

\subsubsection{Models with homothetic symmetry in $4 D$}

The condition of homothetic symmetry in $4 D$ singles out a unique family of metrics, which are generated by the choice $f=\xi^{a /(a+b)}$ (See the Appendix). The homothetic $4 D$ vector as well as the embedding solution in $5 D$ are given by (A-4) and (A-5), respectively. The $4 D$ line element with homothetic symmetry is given by (A-6),

$$
d s^{2}=d t^{2}-\bar{A} t^{2 \alpha k} d x^{2}-\bar{B} t^{2 \beta k} d y^{2}-\bar{C} t^{2 \gamma k} d z^{2}, \quad k \equiv \frac{a}{a+b}=\frac{\alpha+\beta+\gamma}{\alpha^{2}+\beta^{2}+\gamma^{2}} .
$$

We note that the volume of the spatial slices goes like $t^{a k}$. Since $a k>0$, it follows that the volume of space is increasing from an original big-bang at $t=0$. This is regardless of whether some particular direction is contracting. The effective EMT i:5

$$
8 \pi T_{0}^{0}=\frac{k^{2}(\alpha \beta+\alpha \gamma+\beta \gamma)}{t^{2}}, \quad T_{1}^{1}=n_{x} T_{0}^{0}, \quad T_{2}^{2}=n_{y} T_{0}^{0}, \quad T_{3}^{3}=n_{z} T_{0}^{0},
$$

where $n_{x}, n_{y}$ and $n_{z}$ are constants given by

$$
n_{x}=\frac{(-\alpha+\beta+\gamma)}{\alpha+\beta+\gamma}, \quad n_{y}=\frac{(-\beta+\alpha+\gamma)}{\alpha+\beta+\gamma}, \quad n_{z}=\frac{(-\gamma+\alpha+\beta)}{\alpha+\beta+\gamma} .
$$

We note that

$$
n_{x}+n_{y}+n_{z}=1
$$

for any value of $\alpha, \beta$ and $\gamma$. Let us notice some particular cases: (I) If one of the coefficients vanishes, say $\alpha=0$, then $n_{x}=1$ and $n_{y}=-n_{z}$; (II) If two coefficients are equal, say $\alpha=\beta$, then $n_{x}=n_{y}$. In particular, if $\alpha=\beta=0$, then $n_{x}=n_{y}=1$ and $n_{z}=-1$; (III) If $\alpha=\beta=\gamma \neq 0$, then $n_{x}=n_{y}=n_{z}=1 / 3$. The homothetic model exhibits a number of interesting properties, however a detailed study of the matter sources that satisfy (57)-(59) is outside the scope of the present work.

\subsubsection{Perfect fluid with stiff equation of state}

The function $f(\xi)$ in (54) can be determined by imposing some "physical" conditions on the effective picture in $4 D$. If we assume that the effective matter behaves like a perfect fluid, then we obtain

$$
f(\xi)=\left(C_{1} \xi+C_{2}\right)^{m}, \quad \text { where } \quad m \equiv \frac{\alpha+\beta+\gamma}{\alpha^{2}+\beta^{2}+\gamma^{2}+4(\alpha \beta+\alpha \gamma+\beta \gamma)},
$$

and $C_{1}, C_{2}$ are some constants. The effective density $\rho=T_{0}^{0}$ and pressure $p=-T_{1}^{1}=-T_{2}^{2}=-T_{3}^{3}$ in $4 D$ satisfy the stiff equation of state, namely,

$$
\rho=p=\frac{[\gamma(\alpha+\beta)+\alpha \beta] m C_{1}}{8 \pi E \psi^{2} f^{2(\alpha+\beta+\gamma)}} .
$$

\footnotetext{
${ }^{5}$ To simplify the notation, in what follows we suppress the index ${ }^{(4)}$ in ${ }^{(4)} T_{\alpha \beta}$.
} 


\subsubsection{Homothetic $5 D$ embedding for the $4 D$ Kasner spacetime}

For completeness, we should mention that on each spacetime section $\Sigma_{\psi}$ we have

$$
8 \pi T_{00}=\frac{\gamma(\alpha+\beta)+\alpha \beta}{\psi^{2}}\left(\frac{f_{\xi}}{f}\right)^{2} .
$$

Therefore, vacuum solutions in $4 D$ require $\gamma=-\alpha \beta /(\alpha+\beta)$. Then, from the vanishing of $T_{i j}$ we obtain

$$
f(\xi)=\left(C_{1} \xi+C_{2}\right)^{\frac{\alpha+\beta}{\alpha^{2}+\alpha \beta+\beta^{2}}},
$$

where $C_{1}$ and $C_{2}$ are constants of integration. Now, setting $C_{1}=\left[\left(\alpha^{2}+\alpha \beta+\beta^{2}\right) / E(\alpha+\beta)\right]$, we obtain $g_{00}=1$. Thus, the metric in $5 D$ becomes

$$
d S^{2}=d t^{2}-A\left(C_{1} \xi+C_{2}\right)^{2 p_{1}} d x^{2}-B\left(C_{1} \xi+C_{2}\right)^{2 p_{2}} d y^{2}-C\left(C_{1} \xi+C_{2}\right)^{2 p_{3}} d z^{2}-\xi^{2 p_{4}} d \psi^{2},
$$

where

$$
\frac{p_{1}}{\alpha(\alpha+\beta)}=\frac{p_{2}}{\beta(\alpha+\beta)}=-\frac{p_{3}}{\alpha \beta}=\frac{1}{\alpha^{2}+\alpha \beta+\beta^{2}} ; \quad p_{4}=1
$$

We note that

$$
p_{1}+p_{2}+p_{3}=1, \quad p_{1}^{2}+p_{2}^{2}+p_{3}^{2}=1 .
$$

Therefore, (64) can be interpreted as a homothetic $5 D$ embedding for the $4 D$ Kasner spacetime. It is important to point out two things. Firstly, that although (64) represents an empty space in $5 D$, it is not equivalent to the usual 5 -dimensional Kasner space 6 Secondly, it illustrates that the effective EMT in $4 D$, which is given by (51) can vanish even though the induced metric in $4 D$ explicitly depends on the extra coordinate. This is totally different from the isotropic cosmological models, for which the effective EMT is always nonzero when the $5 D$ metric has a explicit dependence on the extra coordinate. In fact, this is a general attribute of models with spatial spherical symmetry [40.

\subsection{Inhomogeneous models: nonstatic vacuum solutions}

Let us now consider the $5 D$ line element (48). In the approach under consideration, the spacetime metric is given by

$$
d s^{2} \equiv d S_{\mid \Sigma_{\psi}}^{2}=g_{\mu \nu} d x^{\mu} d x^{\nu}=E F^{(b / a)} F_{\eta}\left(d t^{2}-\eta^{2} d z^{2}\right)-A F^{2 \alpha} d x^{2}-B F^{2 \beta} d y^{2},
$$

where $F$ is a function of the self-similar variable $\eta=t / z$. These models present two main characteristics. First, they show homothetic symmetry along the $4 D$ vector

$$
\xi_{p}^{\mu}=(t, x, y, z)
$$

where the subindex $p$ indicates that $\xi_{p}^{\mu}$ is the spacetime projection of the $5 D$ homothetic vector $\xi^{A}$ defined in (43). Second, since the spacetime part of the generating $5 D$ metric (48) is independent of $\psi$, it follows that $K_{\mu \nu}=0$ and consequently the effective EMT is

$$
8 \pi T_{\mu \nu}=E_{\mu \nu}=\frac{\Phi_{\alpha ; \beta}}{\Phi}
$$

we recall that the general solution requires $\epsilon=-1$. Therefore,

$$
T \equiv T_{0}^{0}+T_{1}^{1}+T_{2}^{2}+T_{3}^{3}=0
$$

which is a consequence of the fact that $E_{\mu \nu}$ is traceless.

\footnotetext{
${ }^{6}$ We recall the reader that a Kasner space in $5 D$ is given by $d S^{2}=d t^{2}-A t^{2 p_{1}} d x^{2}-B t^{2 p_{2}} d y^{2}-C t^{2 p_{3}} d z^{2}+\epsilon E t^{2 p_{4}} d \psi^{2}$ with $p_{1}+p_{2}+p_{3}+p_{4}=1, \quad p_{1}^{2}+p_{2}^{2}+p_{3}^{2}+p_{4}^{2}=1$.
} 


\subsubsection{Solutions in conventional $4 D$ general relativity}

The nonvanishing components of the EMT are

$$
T_{0}^{0}=-T_{3}^{3}=-\left(\frac{z}{t}\right) T_{3}^{0}=\frac{\gamma\left[\gamma(\alpha+\beta)-\alpha^{2}-\beta^{2}\right]}{8 \pi(\alpha+\beta+\gamma) E}\left[\frac{F_{\eta}}{z^{2} F^{(2 a+b) / a}}\right] .
$$

They can be interpreted as "pancake-like" distributions of matter, with energy flow along the direction of symmetry $z$. A detailed study of the matter distribution (71) is beyond the scope of the present work.

For another interpretation, we note that $T_{\mu \nu}=0$ when

$$
\gamma=\frac{\alpha^{2}+\beta^{2}}{\alpha+\beta}, \quad \alpha \neq-\beta
$$

With this choice, the line element (67) yields a two-parameter family of non-static inhomogeneous vacuum solutions in conventional $4 D$ general relativity. For these solutions, the non-vanishing components of the Riemann tensor in $5 D$ and $4 D$ are all proportional to $(\alpha-\beta) F_{\eta}$. Consequently, for $\alpha \neq \beta$ the vacuum solutions given by (67) and (72) are not equivalent to a $4 D$ Minkowski spacetime and cannot be embedded in a Riemann-flat manifold in $5 D$.

\subsubsection{Vacuum solutions in the Randall-Sundrum (RS2) braneworld scenario}

Without going into deep technical details, in the Randall \& Sundrum braneworld scenario [39] our universe is identified with a fixed singular hypersurface $\Sigma_{\psi_{b}}$ (the brane) embedded in a 5 -dimensional bulk with $\mathbf{Z}_{2}$ symmetry with respect to $\Sigma_{\psi_{b}}$ [37. Due to the presence of matter on the brane, which is described by an EMT that we denote as $\tau_{\mu \nu}$, the extrinsic curvature $K_{\mu \nu}$ is discontinuous across $\Sigma_{\psi_{b}}$. Israel's boundary conditions [41] relate the jump of $K_{\mu \nu}$ to $\tau_{\mu \nu}$, namely, $\left(K_{\mu \nu \mid \Sigma_{\psi_{b}}^{+}}-K_{\mu \nu \mid \Sigma_{\psi_{b}}^{-}}\right)=k\left(\tau_{\mu \nu}-\frac{1}{3} \tau g_{\mu \nu}\right)$, where $k$ is a constant with the appropriate units. Next, $\mathbf{Z}_{2}$ symmetry implies $K_{\mu \nu \mid \Sigma_{\psi_{b}}^{+}}=-K_{\mu \nu \mid \Sigma_{\psi_{b}}^{-}} \equiv K_{\mu \nu}$. Therefore, when the spacetime (the brane) is empty $\left(\tau_{\mu \nu}=0\right)$ it follows that $K_{\mu \nu}=0$. Thus, in braneworld theory the vacuum field equations are obtained from (51) as

$$
{ }^{(4)} G_{\alpha \beta}=8 \pi^{(4)} T_{\alpha \beta}=-\epsilon E_{\alpha \beta},
$$

which means that, in braneworld theory, the effective geometrical matter is $4 D$ is traceless. Therefore, any solution of general relativity with $T=0$ is a solution of the vacuum equations in braneworld.

The conclusion is that (67) can be interpreted as vacuum solutions in the Randall-Sundrum braneworld scenario.

\section{Summary}

Self-similar (homothetic) symmetry seems to be very important in nature7. In cosmological applications, within the context of conventional general relativity in $4 D$, there is a strong evidence that many homogeneous and inhomogeneous cosmological models can be approximated by self-similar models in the asymptotic regimes, i.e., near the initial cosmological singularity and at late times [17]-21]. In order to avoid misunderstanding, it is important to emphasize that in the literature the concept of self-similarity is frequently equated with homothetic symmetry. In this paper we have followed the traditional terminology used in [22]-24, where self-similarity means that the field equations are functions only of a single variable, which in turn allows to reduce them to a system of ordinary differential equations.

Observations indicate that on large scales $(\gg 100 \mathrm{Mpc})$ the universe is homogeneous and isotropic and well described by spatially-flat FRW cosmologies, which are self-similar in $4 D$ and $5 D$. However, there is no reason to expect such features at the early stages of the evolution of the universe. Rather, it is generally accepted that

\footnotetext{
${ }^{7}$ We would like to share with our readers a beautiful quotation about self-similar (homothetic) symmetry, ascribed to Manfred Schroeder, that we learned from a paper by Adrian Popesku [42]: "The unifying concept underlying fractals, chaos and power laws is self-similarity. Self-similarity, or invariance against changes in scale or size, is an attribute of many laws of nature and innumerable phenomena in the world around us. Self-similarity is, in fact, one of the decisive symmetries that shapes our universe and our efforts to comprehend it."
} 
anisotropy could have played a significant role in the early universe and that it has been fading away in the course of cosmic evolution. Therefore, the study of cosmological models that are anisotropic and self-similar appears to be of primary interest.

In this work we have investigated such cosmological models within the context of theories of Kaluza-Klein type, with a large extra dimension. The ladder to go between the physics in $4 D$ and $5 D$ is provided by Campbell-Maagard's embedding theorems, which guarantee that any solution of the $4 D$ Einstein equations of general relativity may be embedded in a solution of the $5 D$ vacuum Einstein equations.

For the case where the $5 D$ metric is diagonal and is a function only of time and the "extra" coordinate $\psi$, we have shown that there are three possible forms for the similarity variable: $\xi=t / \psi ; \zeta=\left(e^{t} / e^{\psi}\right)^{q}$, and $\zeta=\omega_{0} t+k_{0} \psi$. They correspond to three different physical situations, which are described by homothetic, conformal and plane wave-like solutions in $5 D$, respectively. Thus, in the traditional terminology the concept of self-similarity includes conformal and wave-like solutions.

In section 3, we have constructed the most general homothetic and conformal solutions in $5 D$ to the field equations (14)-(19). They are given in terms of one arbitrary function, of the appropriate similarity variable, and three arbitrary parameters, viz., $\alpha, \beta$, and $\gamma$. However, the situation is completely different for the case where the $5 D$ metric is wavelike. This case is more involved and requires a separate discussion.

Following Campbell-Maagard's embedding theorems, the connection to $4 D$ is deduced after choosing an embedding 8 . We have found that the solutions that are homothetic in $5 D$ are relevant to the similarity hypothesis because all the models constructed in $4 D$, by means of dimensional reduction of the metric in $5 D$, exhibit some type of self-similarity. Specifically, we have seen that those that are inhomogeneous and anisotropic inherit the homothetic symmetry from the $5 D$ embedding. The rest of them are either partially homothetic or homothetic along some $4 D$ vector.

Our discussion in section 5 illustrates two important things. Firstly, that when the extra dimension is spacelike the concepts of $4 D$ spatial homogeneity and $4 D$ spatial flatness become totally dependent on the embedding, i.e., on how the coordinates for our 3-dimensional space are chosen. Secondly, that the interpretation in $4 D$ crucially depends on the theory under consideration. In consequence, much work is still needed in order to understand $4 D$ physical models as Lorentzian hypersurfaces in pseudo-Riemannian $5 D$ spaces.

In summary: our work (I) generalizes the $5 D$ embeddings used for the FLRW models; (II) shows that anisotropic cosmologies are, in general, curved in $5 D$, in contrast with FLRW models which can always be embedded in a $5 D$ Riemann-flat (Minkowski) manifold; (III) reveals that anisotropic cosmologies can be curved and devoid of matter, both in $5 D$ and $4 D$, even when the metric in $5 D$ explicitly depends on the extra coordinate, which is quite different from isotropic cosmological models where the effective EMT is always nonzero when the $5 D$ metric has a explicit dependence on the extra coordinate. In fact, this is a general attribute of models with spatial spherical symmetry [40.

To finish this paper, we should mention that from a mathematical point of view the shape of our solutions can be simplified if we introduce "null-like" coordinates (We thank Philippe Spindel for pointing this out [43]). As an illustration, let us consider (29). The $(t-\psi)$ part of that metric can be factorized and written as $E f^{b / a} f_{\xi}(d t+$ $\xi d \psi)(d t-\xi d \psi) \equiv 2 d u d v$. Then, setting $u=f^{(a+b) / a}$ the line element (29) becomes

$$
d S^{2}=2 d u d v-A u^{p_{1}} d x^{2}-B u^{p_{2}} d y^{2}-C u^{p_{3}} d z^{2},
$$

where $p_{1}=2 \alpha a /(a+b), p_{2}=2 \beta a /(a+b)$ and $p_{3}=2 \gamma a /(a+b)$. We note that $\sum_{i=1}^{3}\left(p_{i}-1\right)^{2}=3$. Although in this coordinates the solution looks mathematically simpler than in the original form, its physical interpretation in $4 D$ is elusive since it not clear how to define the hypersurfaces $\Sigma_{\psi}$ orthogonal to the $5 D$ unit vector $\hat{n}^{A}$ along the extra dimension. Therefore, in these coordinates neither the dimensional reduction discussed in Section 5 nor Campbell's theorem can be used for the $4 D$ interpretation of the solutions.

This investigation can be extended, or generalized, in different ways. They follow from the fact that we have not fully examined the possible $4 D$ interpretations of the $5 D$-homothetic solutions (32). Neither the conformal solutions

\footnotetext{
${ }^{8} \mathrm{As}$ it was mentioned in the Introduction, the physically interesting case of finding a $5 D$ embedding for a given $4 D$ spacetime, with a specified physical EMT, is a very difficult task because the effective $4 D$ equations for gravity contain a source term, that is the spacetime projection of the $5 D$ Weyl tensor, which is unknown without specifying the properties of the metric in $5 D[8]$.
} 
(36), nor the plane wave-like solutions induced by the similarity variable (12), have been studied. These are important topics that should be addressed.

\section{Appendix: Homothetic symmetry on $\Sigma_{\psi}$}

Our aim here is to show that the requirement of homothetic symmetry on $\Sigma_{\psi}$ singles out one specific metric in $4 D$. With this aim, let us take the Lie derivative of (54) along the $4 D$ vector

$$
\zeta_{h}^{\mu}=\left(C_{0} t, C_{1} x, C_{2} y, C_{3} z\right),
$$

where $h$ stands for "homothetic" and $C_{0}, C_{2}, C_{3}, C_{3}$ are some constants. We obtain,

$$
\begin{aligned}
\mathcal{L}_{\zeta_{h}} g_{00} & =2 g_{00} C_{0}\left[1+\frac{\xi}{2}\left(\frac{b f_{\xi}}{a f}+\frac{f_{\xi \xi}}{f_{\xi}}\right)\right], \\
\mathcal{L}_{\zeta_{h}} g_{11} & =2 g_{11}\left[C_{1}+\alpha C_{0} \xi\left(\frac{f_{\xi}}{f}\right)\right], \\
\mathcal{L}_{\zeta_{h}} g_{22} & =2 g_{22}\left[C_{2}+\beta C_{0} \xi\left(\frac{f_{\xi}}{f}\right)\right] \\
\mathcal{L}_{\zeta_{h}} g_{33} & =2 g_{33}\left[C_{3}+\gamma C_{0} \xi\left(\frac{f_{\xi}}{f}\right)\right] .
\end{aligned}
$$

The condition $\mathcal{L}_{\zeta_{h}} g_{\mu \nu}=2 g_{\mu \nu}$ requires

$$
\frac{1-C_{1}}{\alpha C_{0}}=\frac{1-C_{2}}{\beta C_{0}}=\frac{1-C_{3}}{\gamma C_{0}} \equiv k, \quad f(\xi) \sim \xi^{k} \quad \text { and } \quad C_{0}=\frac{2 a}{a+k(a+b)},
$$

where $k$ is some constant. Without loss of generality we can set $C_{0}=1$, which implies

$$
k=\frac{a}{a+b}, \quad \text { and } \quad \zeta_{h}^{\mu}=[t,(1-k \alpha) x,(1-k \beta) y,(1-k \gamma) z] .
$$

Thus, the $5 D$ metric

$$
d S^{2}=d t^{2}-\xi^{2 \alpha k} d x^{2}-\xi^{2 \beta k} d y^{2}-\xi^{2 \gamma k} d z^{2}-\xi^{2} d \psi^{2},
$$

generates, on every $\Sigma_{\psi}$, the $4 D$ line element

$$
d s^{2} \equiv d S_{\mid \Sigma_{\psi}}^{2}=d t^{2}-\bar{A} t^{2 \alpha k} d x^{2}-\bar{B} t^{2 \beta k} d y^{2}-\bar{C} t^{2 \gamma k} d z^{2},
$$

which shows homothetic symmetry along $\zeta_{h}^{\mu}$. Here we have introduced the constants $\bar{A}, \bar{B}$ and $\bar{C}$ for dimensional consistency. It should be noted that $\zeta_{h}^{\mu}$ is not the spacetime projection of the $5 D$ vector $\zeta_{(l \neq 1)}^{C}=(t, x, y, z, \psi)$ defined in (43).

\section{References}

[1] J. Bicak, Lecture Notes Phys. 540, 1(2000); gr-qc/0004016

[2] M.A.H. MacCallum, A Century of Relativity Physics (ERE05, the XXVIII Spanish Relativity Meating) Ed. L. Momas and J.D. Alonso, American Institute of Physics (2006); gr-qc/0601102.

[3] S. Yasadjiev, Phys. Rev. D69, 127501(2004); gr-gc/0312019.

[4] S. Rippl, C. Romero and R. Tavakol, Class.Quant.Grav. 12, 2411(1995); gr-qc/9511016.

[5] J.E. Lidsey, C. Romero, R. Tavakol and S. Rippl, Class.Quant.Grav. 14, 865(1997); gr-qc/9907040. 
[6] S.S. Seahra and P.S. Wesson, Class.Quant.Grav. 20 1321(2003); gr-qc/0302015.

[7] P.S. Wesson, "In Defense of Campbell's Theorem as a Frame for New Physics"; gr-qc/0507107.

[8] R. Maartens, Reference Frames and Gravitomagnetism, ed. J Pascual-Sanchez et al. (World Sci., 2001), p93-119; gr-qc/0101059

[9] J. Ponce de Leon, Class.Quant.Grav. 23, 3043(2006); gr-qc/0512067.

[10] J. Ponce de Leon, JCAP 3, 021(2008); arXiv:0802.1953[gr-qc].

[11] K.C. Jacobs, Ph.D Thesis "Bianchi type I cosmological models" (1968-10-01). http://resolver.caltech.edu/CaltechETD:etd-10162002-080822

[12] P. de Bernandis et al, Nature, 404, 955(2000).

[13] H.V.Peiris et al Astrophys.J.Suppl. 148, 213(2003); arXiv:astro-ph/0302225.

[14] D.N. Spergel et al, Astrophys. J. Suppl. 148, 175(2003), arXiv: astro-ph/0302209

[15] W.L. Roque and W.M. Seiler, Gen. Rel. Grav 23, 1151(1991).

[16] J. Ponce de Leon, J. Math. Phys. 32, 3546(1991).

[17] B.J. Carr and A.A. Coley, Gen. Rel. Grav 37, 2165(2005); gr-qc/0508039.

[18] J. Wainwright, M.J. Hancock and C. Uggla C, Class. Quantum Grav. 16 2577(1999), arXiv:gr-qc/9812010.

[19] P.S. Apostolopoulos and M. Tsamparlis, Gen. Rel. Grav. 35, 2051(2003), arXiv:gr-qc/0305017.

[20] P.S. Apostolopoulos, Class.Quant.Grav. 20, 3371(2003), arXiv:gr-qc/0306119

[21] P.S. Apostolopoulos, Class.Quant.Grav. 22, 323(2005), arXiv:gr-qc/0411102.

[22] L.I. Sedov, Similarity and Dimensional Methods in Mechanics (Academic Press, New York, 1959).

[23] M.E. Cahill and A.H. Taub, Commun. Math. Phys. 21, 1(1971).

[24] G. E. Barenblat and Ya. B. Zel'dovich, Ann. Rev. Fluid. Mech. 4, 285(1972).

[25] R. N. Henriksen, A.G. Emslie and P.S. Wesson, Phys. Rev. D 27, 1219(1983).

[26] P.S. Wesson, Phys. Rev. D 34, 3925(1986).

[27] B.J. Carr and A. Yahil, Astrophys. J. 360, 330(1990).

[28] J. Ponce de Leon, Mon. Not. R. astr. Soc. 250, 69(1991).

[29] J. Ponce de Leon, Gen. Rel. Grav. 25, 865(1993).

[30] H. Liu and P.S. Wesson, Int. J. Mod. Phys. D3, 627(1994).

[31] P.S. Wesson, H. Liu and S.S. Seahra, Astron. Astrophys. 358, 425(2000), arXiv:gr-qc/0003012.

[32] G.T. Horowitz, I. Low and A. Zee, Phys. Rev. D62, 0860005(2000), arXiv:hep-th/0004206.

[33] J. Ponce de Leon, Int. J. Mod. Phys. D12, 1053(2003), arXiv:gr-qc/0212036 Gen. Rel. Grav. 36, 923(2004), arXiv:gr-qc/0212058

[34] J. Ponce de Leon, Int. J. Mod. Phys. D 11, 1355(2002); gr-qc/0105120. 
[35] P.S. Wesson, Space-Time-Matter (World Scientific Publishing Co. Pte. Ltd. 1999).

[36] P.S. Wesson and J. Ponce de Leon, J. Math. Phys. 33, 3883(1992).

[37] T. Shiromizu, Kei-ichi Maeda and Misao Sasaki, Phys. Rev. D62, 02412(2000); gr-qc/9910076.

[38] J. Ponce de Leon, Mod.Phys.Lett. A16, 2291(2001), gr-qc/0111011.

[39] L. Randall and R. Sundrum, Phys. Rev. Lett. 83, 4690(1999); hep-th/9906064.

[40] J. Ponce de Leon and P.S. Wesson, J. Math. Phys. 34, 4080(1993).

[41] W. Israel, Nuovo Cim. B44, 1(1966);[Erratum-ibid. B48, 463(1967)].

[42] A. Popesku, "Traffic Self-Similarity" (2001), http://www.bth.se/fou/Forskinfo.nsf

[43] Ph. Spindel, private communication. 